\title{
Natural Deduction for Modal Logic with a Backtracking Operator
}

\author{
Jonathan Payne
}

Received: 14 October 2013 / Accepted: 6 May 2014 / Published online: 23 July 2014

(C) Springer Science+Business Media Dordrecht 2014

\begin{abstract}
Harold Hodes in [1] introduces an extension of first-order modal logic featuring a backtracking operator, and provides a possible worlds semantics, according to which the operator is a kind of device for 'world travel'; he does not provide a proof theory. In this paper, I provide a natural deduction system for modal logic featuring this operator, and argue that the system can be motivated in terms of a reading of the backtracking operator whereby it serves to indicate modal scope. I prove soundness and completeness theorems with respect to Hodes' semantics, as well as semantics with fewer restrictions on the accessibility relation (Hodes restricts his attention to S5).
\end{abstract}

Keywords Modal logic $\cdot$ Backtracking $\cdot$ Natural deduction

\section{Introduction}

Harold Hodes in [1] introduces an extension of first-order modal logic featuring a backtracking operator ' $\downarrow$ '. The purpose of this operator is similar to that of an actuality operator. But, instead of exempting what follows from the scope of all enclosing modal operators, it exempts it only from the innermost modal operator. Or, in terms of the possible worlds semantics, instead of causing a formula to be evaluated at some specified 'actual' world, the backtracking operator causes a formula to be evaluated at the 'last visited' world, so to speak.

J. Payne $(\bowtie)$

Institute of Philosophy, University of London, Senate House, Malet Street, London, WC1E 7HU, UK e-mail: jonathan.payne@sas.ac.uk 
Hodes gives a semantics (which I give an overview of in Section 2), but does not supply a proof theory. ${ }^{1}$ This semantics reflects a reading of the operator as one which allows more flexible 'travel' through worlds in evaluating the truth value of a formula. If the semantics of $\square$ and $\diamond$ are thought of as instructions to travel to an accessible world in which the truth value of a formula is evaluated, Hodes' semantics for $\downarrow$ gives instructions to travel back to the world most recently passed through, to evaluate the truth value of a formula there. A useful comparison is to the actuality operator. The usual semantics for an actuality operator gives directions to evaluate the truth value of a formula at a privileged possible world in the model- the actual world.

But, as mentioned, there is another-more syntactic-reading of the operator, as a scope indicator. That is, the operator simply indicates that what follows it is to be exempt from the scope of the innermost modal operator. Again, compare with the actuality operator; this may be taken to be an indicator that what follows is to be evaluated as exempt from the scope of all enclosing modal operators. Thus, the role of $\downarrow$ is akin to that of parentheses, yet allowing for more nuanced scope distinctions. The aim of this paper is to provide a proof system for modal logics featuring the operator, which I will claim does justice to this reading; the proof theory gives rules for 'looking inside' the scope of a modal operator, and then, when the $\downarrow$ operator is encountered, pulling the appended formula out of that scope.

As well as explaining this alternative reading of the operator, such a proof theory may be desirable from the point of view of certain philosophical or other uses of modal logic. For example, extensions of modal logic may be desired to gain expressive power without committing oneself to quantifying over possible worlds (or their analogues) or to the members of domains of possible worlds. In some cases, modal logic may be introduced specifically for the purposes of avoiding quantification over some entities or other. A proof theory would allow somebody not to rely on the semantics to give sense to claims involving $\downarrow .^{2}$

Nonetheless, even if one is not persuaded of the need for a proof theory for such purposes, the fact that the operator appears to admit of the syntactic reading should be motivation enough to develop a proof theory which represents such a reading.

Before presenting the proof system, in Section 2 I will give an overview of the semantics which Hodes provides for the operator, albeit with a few minor differences. In Section 3 I present a natural deduction system for the operator for propositional modal logic featuring the operator, which makes uses of labelling each line of a proof. In Section 4, I motivate the natural deduction system in terms of the scope exemption reading of the operator. In Section 5 I prove that the deduction system is sound and complete with respect to models whose accessibility relation is serial. Finally, in Section 6 I discuss strengthening the logic and prove corresponding soundness and completeness theorems for restricted classes of models.

\footnotetext{
${ }^{1} \mathrm{He}$ does refer to an unpublished manuscript featuring a proof theory, but there is none published.

${ }^{2}$ For recent examples of philosophical uses of the backtracking operator, or operators similar to it, see [2, 3, 6-8]. For at least some of these uses, it would be desirable to explain the meaning of the operator in terms of scope exemption rather than world travel.
} 


\section{Semantics}

Let $\mathscr{L}_{\downarrow}$ be a typical language for propositional modal logic; it consists of countably many propositional variables $p, q, r, \ldots$, connectives $\wedge$ and $\neg$ and a necessity operator $\square$. In addition, it shall have an additional sentential operator $\downarrow$, called the backtracking operator. The intended effect of the backtracking operator will be to exempt what follows it from the scope of the innermost modal operator from which it is not already exempt (so, for example, $p, \square \downarrow p$ and $\square \square \downarrow \downarrow p$ should all be counted as equivalent). Other sentential connectives $\vee, \rightarrow$ and a possibility operator $\diamond$ can be defined in the usual way.

The semantics presented here is essentially that of [1], with the main differences being: (a) Hodes' semantics is for quantified modal logic, whereas I will only describe the case for propositional modal logic (I discuss extending to quantified modal logic briefly in Section 7). (b) Hodes' logic is an extension of S5, so that the accessibility relation is an equivalence, whereas the only restriction on the equivalence relation here is that it is serial. (c) Hodes only defines satisfaction for a certain class of formulas, whereas the semantics presented here places no such restriction.

A model is a triple $\mathscr{M}=\langle W, R, a\rangle$, where $W$ is a set (of possible worlds), $R \subseteq$ $W \times W$ is the accessibility relation, and $a$ is an assignment function which assigns to each propositional variable $p$ at a world $w \in W$ a truth value $a(w, p) \in\{T, F\}$.

Only one restraint will be placed on the accessibility relation for now, and that is that it is serial. So, for any $w \in W$ there is a $w^{\prime} \in W$ such that $w R w^{\prime}$.

Then, a satisfaction relation is defined, not for each world, but for each finite sequence of worlds of the appropriate type. So, we first make the following definition:

Definition 1 Given a model $\mathscr{M}$, a world sequence is a member of the following set:

$$
\mathrm{WS}_{\mathscr{M}}=\left\{\left\langle w_{1}, \ldots, w_{k}\right\rangle: k \geq 1, \forall i \leq k, w_{i} \in W \text { and } \forall i<k, w_{i} R w_{i+1}\right\}
$$

As a result of seriality, for every world sequence there will be a world sequence extending it (and so there are world sequences of arbitrary length). The sequence of worlds at which a formula is evaluated may be thought of as a kind of memory, which keeps track of which worlds have been travelled through.

Some terminology for members of WS $\mathscr{M}$ will be useful. I shall write $\mathbf{w}$ for an arbitrary member of $\mathrm{WS}_{\mathscr{M}}$. Where $\mathbf{w}=\left\langle w_{1}, \ldots, w_{k}\right\rangle$, then:

$$
\begin{aligned}
\left\langle\mathbf{w}, w^{\prime}\right\rangle & =\left\langle w_{1}, \ldots, w_{k}, w^{\prime}\right\rangle \\
\mathbf{w}- & = \begin{cases}\left\langle w_{1}, \ldots, w_{k-1}\right\rangle, & k>1 \\
\left\langle w_{1}\right\rangle, & k=1\end{cases} \\
t(\mathbf{w}) & =w_{k} \\
l(\mathbf{w}) & =k
\end{aligned}
$$


Finally, if $\mathbf{w}=\left\langle w_{1}, \ldots, w_{k}\right\rangle$ and $\mathbf{w}^{\prime}=\left\langle w_{1}, \ldots, w_{l}\right\rangle$ for $l<k$, then I shall write that $w$ extends (or is an extension of) $w^{\prime}$, and that $w^{\prime}$ truncates (or is a truncation of) $w$.

Now, we can define satisfaction of a formula at a world sequence as follows:

Definition 2 Let $\phi$ be a formula of $\mathscr{L}_{\downarrow}, \mathscr{M}$ a model, and $\mathbf{w} \in \mathrm{WS} \mathscr{\mathscr { M }}$. Then, $\mathscr{M}, \mathbf{w} \models$ $\phi$ is defined inductively as follows:

Where $\phi$ is a propositional variable $p$ :

$$
\mathscr{M}, \mathbf{w}=p \text { iff } a(t(\mathbf{w}), p)=T
$$

For propositional connectives:

$$
\begin{gathered}
\mathscr{M}, \mathbf{w} \models \neg \phi \text { iff } \mathscr{M}, \mathbf{w} \not \models \phi \\
\mathscr{M}, \mathbf{w}=\phi \wedge \psi \text { iff } \mathscr{M}, \mathbf{w} \models \phi \text { and } \mathscr{M}, \mathbf{w} \models \psi
\end{gathered}
$$

For modal operators (including $\downarrow$ ):

$$
\begin{aligned}
& \mathscr{M}, \mathbf{w} \models \square \phi \text { iff for all } w^{\prime} \text { s.t. } t(\mathbf{w}) R w^{\prime}, \mathscr{M},\left\langle\mathbf{w}, w^{\prime}\right\rangle \models \phi \\
& \mathscr{M}, \mathbf{w} \models \downarrow \phi \text { iff } \mathscr{M}, \mathbf{w} \models \phi
\end{aligned}
$$

Note that, although this definition is only given for when $\mathbf{w} \in \mathrm{WS}_{\mathscr{M}}$, there is nothing which requires this, rather than that $\mathbf{w}$ is any sequence of worlds. But, given the clause for $\square$, it will only ever be $\mathbf{w} \in$ WS which are relevant. Later on, however (Section 6), it will be necessary to consider such an extension. $\phi$.

Where $\mathscr{M}$ is clear from context, I will sometime write $\mathbf{w} \models \phi$ in place of $\mathscr{M}, \mathbf{w} \models$

A consequence relation can then be defined:

Definition 3 Where $\Gamma$ is a set of formulas in $\mathscr{L}_{\downarrow}$, and $\phi$ a formula of $\mathscr{L}_{\downarrow}$, then $\Gamma \models \phi$ iff: For all $\mathscr{M}$ and $w \in W_{\mathscr{M}}$, if $\mathscr{M},\langle w\rangle \models \psi$ for each $\psi \in \Gamma$, then $\mathscr{M},\langle w\rangle \models \phi$.

\section{Proof Theory}

In this section I shall describe a natural deduction system for the logic. This will consist of an introduction and elimination rule for each connective and operator, and a definition of the notion of a deduction.

An important feature will be that deductions and inference rules will operate on labelled formulas. A labelled formula is a pair $\phi$; $\mathbf{s}$, where $\phi$ is a formula of $\mathscr{L}_{\downarrow}$, and $\mathbf{s}$ is a (possibly empty) finite sequence of natural numbers. Where the label is empty, 
I shall write $\phi ;-$. The same terminology as for world sequences will be used, with the exception that for labels, where $\mathbf{s}=\left\langle n_{1}, \ldots, n_{k}\right\rangle$ I shall write:

$$
\mathbf{s}-= \begin{cases}\left\langle n_{1}, \ldots, n_{k-1}\right\rangle, & k>0 \\ \langle\rangle, & k=0\end{cases}
$$

The rules are as follows:

$$
\begin{array}{ccc}
(\wedge-\mathrm{I}) \frac{\phi ; \mathbf{s}}{\phi \wedge \psi ; \mathbf{s}} & \left(\wedge-\mathrm{E}_{1}\right) \frac{\phi \wedge \psi ; \mathbf{s}}{\phi ; \mathbf{s}} \quad\left(\wedge-\mathrm{E}_{2}\right) \frac{\phi \wedge \psi ; \mathbf{s}}{\psi ; \mathbf{s}} \\
{[\phi ; \mathbf{s}]} & {[\neg \phi ; \mathbf{s}]} \\
\vdots & \vdots \\
(\neg-\mathrm{I}) \frac{q \wedge \neg q ; \mathbf{t}}{\neg \phi ; \mathbf{s}} & (\neg-\mathrm{E}) \frac{q \wedge \neg q ; \mathbf{t}}{\phi ; \mathbf{s}} \\
(\square-\mathrm{I}) \frac{\phi ; \mathbf{s}}{\square \phi ; \mathbf{s}-} & (\square-\mathrm{E}) \frac{\square \phi ; \mathbf{s}}{\phi ;(\mathbf{s}, n\rangle}
\end{array}
$$

(With a restriction on the ( $\square$-I) rule that $\phi$; s may only depend on assumptions with labels $\mathbf{t}$ which are truncations of $\mathbf{s}$.)

$$
(\downarrow-I) \frac{\phi ; \mathbf{s}}{\downarrow \phi ; \mathbf{s}, n\rangle} \quad(\downarrow-E) \frac{\downarrow \phi ; \mathbf{s}}{\phi ; \mathbf{s}-}
$$

With these rules, rules for the defined connectives and operators can be deduced. In particular, rules for $\diamond$ will be:

$$
\begin{gathered}
{[\phi ;\langle\mathbf{s}, n\rangle]} \\
\vdots \\
(\diamond-\mathrm{I}) \frac{\phi ;\langle\mathbf{s}, n\rangle}{\diamond \phi ; \mathbf{s}} \quad(\diamond-\mathrm{E}) \frac{\diamond \phi ; \mathbf{s}}{\psi ; \mathbf{t}} \quad \psi \quad
\end{gathered}
$$

(with no restriction on the introduction rule, and a restriction on the elimination rule that $\mathbf{t}$ is either $\mathbf{s}$ or a truncation of $\mathbf{s}$ ).

Since deductions in the system will involve discharging assumptions and restrictions on which assumptions are allowed, the notion of a deduction rule corresponding to each inference rule-which will specify how assumptions are to be discharged-is needed (c.f. [4]). Deduction rules are $n$-tuples of the form $\left\langle\left\langle\Gamma_{1}, \theta_{1} ; \mathbf{s}_{1}\right\rangle, \ldots,\left\langle\Gamma_{k}, \theta_{k} ; \mathbf{s}_{k}\right\rangle\right\rangle$, which say that when $\theta_{1} ; \mathbf{s}_{1}, \ldots \theta_{k-1} ; \mathbf{s}_{k-1}$ have been derived using undischarged assumptions $\Gamma_{1} \ldots \Gamma_{k-1}$ respectively, then $\phi_{k} ; \mathbf{s}_{k}$ can be derived with undischarged assumptions $\Gamma_{k}$. For example, the deduction rule corresponding to ( $\neg-\mathrm{I})$ will be $\langle\langle\Gamma, p \wedge \neg p ; \mathbf{t}\rangle,\langle\Gamma \backslash\{\phi\}, \neg \phi ; \mathbf{s}\rangle\rangle$. The deduction rules can simply be read off the rules as presented here.

Two notions of proof-theoretic consequence can then be defined-one for labelled formulas, and one for unlabelled formulas. Since we are interested in deductions between formulas of $\mathscr{L}_{\downarrow}$, and since such formulas are unlabelled, then strictly speaking, the relation between labelled formulas is not one of provability. It is rather simply 
a stepping stone towards the provability relation between (unlabelled) formulas. This latter relation is what we are primarily interested in.

Definition 4 Let $\Delta$ be a set of labelled formulas of $\mathscr{L}_{\downarrow}$, and $\phi ; \mathbf{s}$ a labelled formula of $\mathscr{L}_{\downarrow}$. Then $\Delta \vdash \phi ; \mathbf{s}$ iff there is some sequence of ordered pairs:

$$
\left\langle\left\langle\Delta_{1}, \theta_{1} ; \mathbf{t}_{1}\right\rangle, \ldots,\left\langle\Delta_{n}, \theta ; \mathbf{t}_{n}\right\rangle\right\rangle
$$

such that:

- $\Delta_{n}=\Delta$ and $\theta_{n} ; \mathbf{t}_{n}$ is $\phi ; \mathbf{s}$

- For $i \leq n$, either:

- $\theta_{i} ; \mathbf{t}_{i} \in \Delta_{i}$, or

- There are $j, k<i$ such that $\left\langle\left\langle\Delta_{j}, \theta_{j} ; \mathbf{t}_{j}\right\rangle,\left\langle\Delta_{k}, \theta_{k} ; \mathbf{t}_{k}\right\rangle,\left\langle\Delta_{i}, \theta_{i} ; \mathbf{t}_{i}\right\rangle\right\rangle$ is an instance of one of the deduction rules corresponding to the inference rules.

Definition 5 Where $\Gamma$ is a set of (unlabelled) formulas of $\mathscr{L}_{\downarrow}$, and $\phi$ a formula of $\mathscr{L}_{\downarrow}$, then $\Gamma \vdash \phi$ iff $\Gamma^{*} \vdash \phi ;-$, where $\Gamma^{*}$ is the set of labelled formulas resulting from replacing each $\psi \in \Gamma$ by $\psi$; - (i.e. $\psi$ together with an empty label).

\section{Motivation}

Before proving that the inference rules given here are both sound and complete with respect to the semantics, I would first like to say more about the motivation behind the proof theory. There are two aims which I have. The first concerns a worry which may be had if the proof theory is wanted in order to avoid reliance on the possible worlds semantics for more than pragmatic reasons. It might be worried that, due to the presence of labels - which it is tempting to take as referring to worlds or sequences of worlds - the proof theory does not succeed in avoiding reliance on the possible worlds semantics. Secondly, I claimed before that the natural deduction system can be seen as explaining the reading of the $\downarrow$ operator as exempting formulas from the scope of other operators. Here I will argue for that claim.

One way in which we may try to motivate the proof system is by reference to the semantics. On this view, a labelled formula is a formula of a kind of extended language, and the labels are something like variables referring to sequences of worlds. Then, a formula (of the extended language) $\phi$; $\mathbf{s}$ makes the claim that $\phi$ is true at the sequence of worlds $\mathbf{s}$. The inference rules then aim to capture certain valid inferences in this language. A soundness theorem will then be an essential part of the motivation of the proof theory, in that it will show that the inference rules are indeed valid inference rules - that is, they are truth preserving in the sense of the semantics. (This will also require an extension of the semantics given in Section 2 so that labelled formulas are given satisfaction conditions.)

But given one motivation for developing a proof theory, this will not do. There are potential philosophical applications of such a proof theory whose aim is to 
avoid reference to, and quantification over, worlds. That is, they wish to relegate the semantics to a purely secondary, pragmatic role. But if the proof theory is ultimately motivated by the semantics, then this can not be the case. In any case, such a motivation would fail to shed light on the scope exemption reading of the $\downarrow$ operator.

So, instead, we wish to have a motivation for the proof theory which derives from this scope exemption reading of the operator. Then, a soundness theorem will not play a role in motivating the proof theory in light of the semantics, but will rather play the role-along with a completeness theorem — of motivating the pragmatic value of the semantics. For, given a soundness theorem, one will be able to use the semantics for useful ends, in proving that a certain formula is not derivable from other formulas, and so on.

How might such a motivation look? Firstly, labels must not be thought of as referring to worlds. Indeed, it should be borne in mind that they are not part of the language at all. They are merely part of the proof theory, and can be explained as a kind of bookkeeping device, not dissimilar to the use of line numbers, the lists of undischarged assumptions which are common in many ways of laying out formal proofs, or even to the various horizontal and vertical lines which appear in many ways of laying out proofs. It is perhaps better to think, not of labelled formulas, but of labelled lines (it just happens that it is simpler for metatheoretical purposes to treat labels as attaching to formulas).

If labels are not part of the language, then there can be no danger that they refer to anything in the semantics (just as line numbers and the like do not). Indeed, labelled formulas are not the kind of thing that can be asserted, or the kind of thing that have truth-conditions or satisfaction-conditions. Since labelled formulas are not the kind of thing that can be true or false, and the inference rules are relations between labelled formulas, it follows that the inference rules can not be motivated in terms of validity (i.e. necessary truth preservation). A different motivation is thus required.

The main rules which need motivating are the $\square$ and $\downarrow$ rules. These can be motivated, not in terms of validity, but rather as rules for temporarily ignoring, and then reinstating, modal operators, whilst the labels serve as a reminder as to when a modal operator is being ignored. So, rather than serving as a memory of worlds, $\mathbf{s}$ serves as a memory of modal scope.

Consider first the rules for $\square$, and in particular $\square$-E. This says that, given a formula $\square \phi$, we may temporarily ignore the outermost $\square$, as long as we make a note that a necessity operator is being ignored, and that we must eventually reinstate it. This note is the label. So, we have the inference:

1. $\square \phi$

2. $\phi ; 0$

The $\square$-I rule then does the reverse. It tells us that, if we have a label signifying that a necessity operator is being ignored (and that this label was not introduced in an assumption), then we may reinstate the necessity operator at the same time as removing the label. For example, the above proof could be continued as follows:

\section{3. $\square \phi$}

Getting us back to where we started. 
The rules for the $\downarrow$ operator tell us what we can do when we encounter that operator within the scope of a necessity operator-i.e. when we encounter that operator on a line with a label. In that case, the intended interpretation of the $\downarrow$ operator is that it exempts what follows from the scope of the necessity operator. Thus, we can remove the label at the same time as removing the $\downarrow$ operator. I.e., we are permitted to permanently ignore the necessity operator, since the backtracking operator cancels out the scope.

For example, if, in the above case, $\phi$ is $\downarrow \psi$, then we may continue the proof as follows:
2. $\downarrow \psi ; 0$
3. $\psi$

Finally, the $\downarrow$-I rule allows us to do the reverse. That is, we can append $\downarrow$ to a formula, as long as we also make a note that we are required to encapsulate the resulting formula in a necessity operator in order to cancel the backtracking operator. Taken together with the $\square$-I rule, this allows us to produce the converse of the above proof that $\square \downarrow \psi$ entails $\psi$.

The rules for connectives, which do not alter the labels in any way, simply tell us that we can manipulate these connectives in the usual way, even if we are temporarily ignoring the scope of a necessity operator.

There is one final comment that is required in order to justify the proof theory, and that concerns the specific form (or perhaps, the specific structure) that the labels take. Labels must be able to carry information about how many modal operators are being ignored, so as to deal with nested operators. So, for example, comparable to the inference above from $\square \downarrow \psi$ to $\psi$, we can give the following inference from $\square \square \downarrow \downarrow \psi$ to $\psi$ :

1. $\square \square \downarrow \downarrow \psi ;-$

2. $\square \downarrow \downarrow \psi \quad ; 0$

3. $\downarrow \psi ; 00$

4. $\downarrow \psi \quad ; 0$

5. $\psi \quad ;-$
(Assumption)

The appearance of a label with length 2 on line 3 tells us that, at that point, we are ignoring two nested modal operators.

So, the length of a label measures the number of operators currently being ignored. But, if we just need to measure that, then why do we need to do it with a sequence of natural numbers, rather than a single natural number (in which case it would be the number itself, rather than the length of a sequence, which does the measuring)? The reason is that there may be need, considering the restriction on the $\square$-I rule, to introduce two assumptions with different labels but of the same length. 
For example, consider the derived $(\diamond-\mathrm{E})$ rule:

$$
\begin{gathered}
{[\phi ;\langle\mathbf{s}, n\rangle]} \\
\vdots \\
(\diamond-\mathrm{E}) \frac{\diamond \phi ; \mathbf{s} \quad \psi ; \mathbf{t}}{}
\end{gathered}
$$

Where $\mathbf{t}$ is either $\mathbf{s}$ or a truncation of $\mathbf{s}$ (this restriction follows from the restriction on the ( $\square$-I) rule). Now, it must be possible to introduce separate labels of the form $\langle\mathbf{s}, n\rangle$ if using this rule more than once at a time. For example, to prove $\diamond \phi \vee \diamond \psi \rightarrow$ $\diamond(\phi \vee \psi)$, two such subproofs are needed. ${ }^{3}$ To differentiate the two subproofs, we must use distinct labels of length 1 .

So, the proof theory can be motivated on purely syntactic grounds, without reference to the semantics. Motivated in this way, the semantics may still however by useful, but that is not because it it required to motivate the deduction system. Rather, it is because a model of $\phi$ can be used to show that $\phi$ is consistent, or a model of $\Gamma \cup\{\neg \phi\}$ can be used to show that we have no hope of proving $\phi$ from $\Gamma$. Of course, for the semantics to be useful for such purposes, a soundness theorem is required. The aim of the next section is to prove such a theorem (along with a completeness theorem).

\section{Soundness and Completeness}

\subsection{Soundness}

In this section we shall prove the soundness of $\vdash$ with respect to $\models$. That is, that for any set of formulas $\Gamma$ and formula $\phi$, if $\Gamma \vdash \phi$ then $\Gamma \models \phi$.

This will go by way of defining a model-theoretic consequence relation for labelled formulas, analogous to the corresponding proof-theoretic consequence relation. Before defining this, we shall need to define a certain type of mapping from labels to (possibly empty) world sequences, which preserves certain parts of the structure of the sequences.

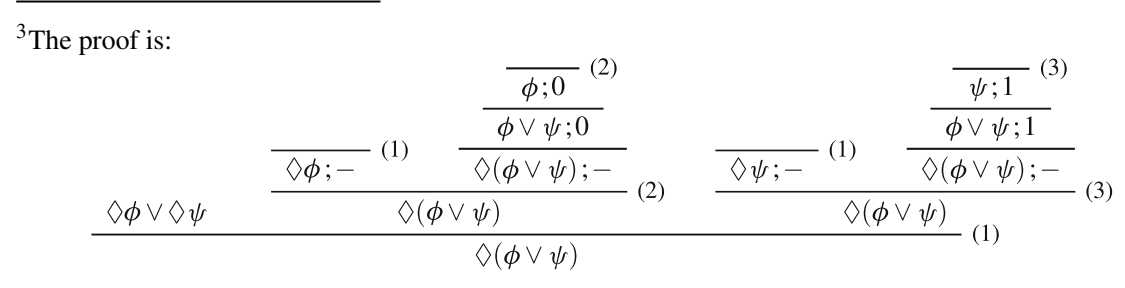

(which makes use of derived rules for $\vee$ as well). 
Definition 6 A function $f: \mathbb{N}^{<\omega} \rightarrow$ WS $\mathscr{M} \cup\{\langle\rangle\}$ is a homomorphism iff, for all $\mathbf{s}, \mathbf{t} \in \mathbb{N}^{\omega}$ :

- $\quad$ s properly extends $\mathbf{t} \Leftrightarrow f(\mathbf{s})$ properly extends $f(\mathbf{t})$

$-\quad l(f(\mathbf{s}))=l(\mathbf{s})$.

Denote the set of homomorphisms for a model $\mathscr{M}$ by $\operatorname{Hom}_{\mathscr{M}}$.

There are a few important consequences of this definition:

\section{Proposition 1}

1. For any model $\mathscr{M}, \operatorname{Hom}_{\mathscr{M}} \neq \emptyset$.

2. For any $f \in \operatorname{Hom}_{\mathscr{M}}$, $\mathbf{s}$ and $n, f(\langle\mathbf{s}, n\rangle)=\left\langle f(\mathbf{s}), w^{\prime}\right\rangle$ for some $w^{\prime}$ such that $w R w^{\prime}$.

3. If $l(\mathbf{s})>0$ then $\langle w, f(\mathbf{s})\rangle=\langle w, f(\mathbf{s}-)\rangle$.

\section{Proof}

1. Since models are serial, there is an infinite sequence of worlds $\left\langle w_{1}, w_{2}, \ldots\right\rangle$ such that $w_{i} R w_{i+1}$ for all $i \in \mathbb{N}$. Now, simply define $f(\mathbf{s})=\left\langle w_{1}, \ldots, w_{l(\mathbf{s})}\right\rangle$. It is simple to check that this satisfies the required properties.

2. First, note that $l(f(\mathbf{s}, n))=l(\mathbf{s}, n)=l(\mathbf{s})+1=l(f(\mathbf{s}))+1$ by the second condition. Moreover, by the first condition, $f(\mathbf{s}, n)$ extends $f(\mathbf{s})$. Hence, we must have some $w$ such that $f(\mathbf{s}, n)=\langle f(\mathbf{s}), w\rangle$. The accessibility requirement follows from the fact that $f(\mathbf{s}, n) \in \mathrm{WS} \mathscr{M}$.

3. Suppose $l(\mathbf{s})>0$, so that $\mathbf{s}=\left\langle n_{1}, \ldots, n_{k}\right\rangle$ for some $k>0$, and so $f(\mathbf{s})=$ $\left\langle w_{1}, \ldots, w_{k}\right\rangle \in \mathrm{WS}$ (by the second condition).

Now, $f(\mathbf{s}-)=\left\langle w_{1}, \ldots, w_{k-1}\right\rangle$, since $l(f(\mathbf{s}))=k-1$ and $f(\mathbf{s})$ extends $f(\mathbf{s})$. (Note: $\left\langle w_{1}, \ldots, w_{k-1}\right\rangle$ may not be the same as $f(\mathbf{s})-$. In particular, this would not be the case when $k=1$.)

Thus, $\langle w, f(\mathbf{s})\rangle=\left\langle w, w_{1}, \ldots, w_{k-1}\right\rangle=\langle w, f(\mathbf{s})\rangle-$ as required.

We are now in a position to define an analogue of consequence for labelled formulas. But first, we need to define a notion of satisfaction for labelled formulas:

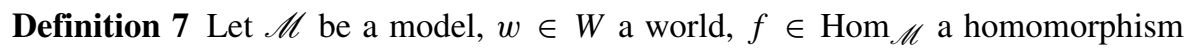
and $\phi ; \mathbf{s}$ a labelled formula. Then $\mathscr{M}, w, f \models \phi ; \mathbf{s}$ iff $\mathscr{M},\langle w, f(\mathbf{s})\rangle \models \phi$.

This can then be extended to sets of labelled formulas in the obvious way:

Definition 8 Let $\mathscr{M}$ be a model, $w \in W$ a world, $f \in$ Hom $_{\mathscr{M}}$ a homomorphism and $\Delta$ a set of labelled formulas. Then $\mathscr{M}, w, f \models \Delta$ iff for all $\phi ; \mathbf{s} \in \Delta, \mathscr{M}, w, f \models$ $\phi ; \mathbf{s}$.

We can then put these together to define consequence for labelled formulas: 
Definition 9 Let $\Delta$ be a set of labelled formulas, $\phi ; \mathbf{s}$ a labelled formula. Then, $\Delta \models \phi ; \mathbf{s}$ iff for every $\mathscr{M}, w \in W_{\mathscr{M}}$ and homomorphism $f \in \operatorname{Hom}_{\mathscr{M}}$,

$$
\text { if } \mathscr{M}, w, f \models \Delta \text { then } \mathscr{M}, w, f \models \phi \text {; } \mathbf{s}
$$

We can now state and prove a soundness theorem with respect to this notion of consequence, and use this to prove a soundness theorem with respect to the notion of consequence for unlabelled formulas (Definition 3).

Proposition 2 Let $\Delta$ be a set of labelled formulas and $\phi$; $\mathbf{s}$ a labelled formula. Then

$$
\text { If } \Delta \vdash \phi ; \mathbf{s} \text { then } \Delta \models \phi ; \mathbf{s}
$$

Proof First, it needs to be checked that each deduction rule is sound. That is, when $\left\langle\left\langle\Gamma_{1}, \theta_{1} ; \mathbf{s}_{1}\right\rangle, \ldots,\left\langle\Gamma_{k}, \theta_{k} ; \mathbf{s}_{k}\right\rangle\right\rangle$ is an instance of a deduction rule, then if $\Gamma_{i}=\theta_{i} ; \mathbf{s}_{i}$ for each $i<k$, then $\Gamma_{k} \models \theta_{k} ; \mathbf{s}_{k}$.

That this is so for the propositional connectives is standard (since the labels do not really play a role). It can be proved for the rules for operators as follows:

$(\downarrow-\mathbf{I})$ : The deduction rule for $(\downarrow-\mathrm{I})$ is $\langle\langle\Gamma, \phi ; \mathbf{s}\rangle,\langle\Gamma, \downarrow \phi ; \mathbf{s}, n\rangle\rangle$. Suppose that $\Gamma \models$ $\phi$; s, so that for any $w, f$, if $w, f \models \Gamma$, then $w, f(\mathbf{s}) \models \phi$. Now, consider $w, f$ such that $w, f \models \Gamma$, and we wish to show that $w, f(\mathbf{s}, n) \models \downarrow \phi$. Since $f$ is a homomorphism, $f(\mathbf{s}, n)=f(\mathbf{s}), w^{\prime}$ for some $w^{\prime}$ such that $t(f(\mathbf{s})) R w^{\prime}$. So, $w, f(\mathbf{s}, n) \models \downarrow \phi$ iff $w, f(\mathbf{s}), w^{\prime} \models \downarrow \phi$ iff $w, f(\mathbf{s}) \models \phi$, which we already have.

$(\downarrow-\mathbf{E})$ : The deduction rule is $\langle\langle\Gamma, \downarrow \phi ; \mathbf{s}\rangle,\langle\Gamma, \phi ; \mathbf{s}-\rangle\rangle$. There are two cases to consider: firstly, when $\mathbf{s}$ is empty, so that $\mathbf{s}-$ is also empty, and secondly when $\mathbf{s}$ is not empty.

Consider the first case. Suppose that $\Gamma \models \downarrow \phi$; - so that for any $w, f$, if $w, f \models \Gamma$ then $\langle w\rangle \models \downarrow \phi$ (since $\mathbf{s}$ is empty, $f$ can be safely ignored). Now consider some $w, f$ such that $w, f \models \Gamma$ and so $\langle w\rangle \models \downarrow \phi$. So, by the definition of $\downarrow,\langle w\rangle \models \phi$, which is as required.

Suppose that $\mathbf{s}$ is not empty. Suppose $\Gamma \models \downarrow \phi$; $\mathbf{s}$ so that for all $w, f$, if $w, f \models \Gamma$ then $w, f(\mathbf{s}) \models \downarrow \phi$. Now consider $w, f$ such that $w, f \models \Gamma$, so $w, f(\mathbf{s}) \models \downarrow \phi$. By definition of $\downarrow,(w, f(\mathbf{s}))-\models \phi$. But, since $l(\mathbf{s})>0,(w, f(\mathbf{s}))=w, f(\mathbf{s})$. So $w, f(\mathbf{s}) \models \phi$ which is what is required.

( $\square$-E): The deduction rule is $\langle\langle\Gamma, \square \phi ; \mathbf{s}\rangle,\langle\Gamma, \phi ; \mathbf{s}, n\rangle\rangle$. Suppose that $\Gamma \models \square \phi ; \mathbf{s}$, so that for all $w, f$, if $w, f \models \Gamma$ then $w, f(\mathbf{s}) \models \square \phi$. Now consider $w, f$ such that $w, f \models \Gamma$, and we wish to show that $w, f(\mathbf{s}, n) \models \phi$. By the properties of $f$, $f(\mathbf{s}, n)=f(\mathbf{s}), w^{\prime}$ for some $w^{\prime}$ such that $w R w^{\prime}$. But, since $w, f(\mathbf{s}) \models \square \phi$ and by the definition of $\square$, for any $w^{\prime}$ such that $w R w^{\prime}, w, f(\mathbf{s}), w^{\prime} \models \phi$. So, $w, f(\mathbf{s}, n) \models \phi$ as required.

( $\square$-I): The deduction rule is $\langle\langle\Gamma, \phi ; \mathbf{s}\rangle,\langle\Gamma, \square \phi ; \mathbf{s}\rangle\rangle$ with the restriction that if $\psi ; \mathbf{t} \in$ $\Gamma$ then s properly extends $\mathbf{t}$. Suppose that $\Gamma \models \square \phi$; s for such a $\Gamma$. So, for any $w, f$, if $w, f \models \Gamma$ then $w, f(\mathbf{s}) \models \phi$.

Now, consider $w, f$ such that $w, f \models \Gamma$. We wish to show that $w, f(\mathbf{s})=\square \phi$. Suppose not, then for some $w^{\prime}$ such that $t(f(\mathbf{s})) R w^{\prime}, w, f(\mathbf{s}), w^{\prime} \not \models \phi$. 
Now, it is clear that a homomorphism $f^{\prime}$ can be defined such that:

- $\quad f^{\prime}(\mathbf{t})=f(\mathbf{t})$ for all $\mathbf{t}$ which s properly extends

- $f^{\prime}(\mathbf{s})=f(\mathbf{t}-), w^{\prime}$

and allowed to take any other permitted value elsewhere.

So, $w, f^{\prime}(\mathbf{s}) \not \models \phi$. So, $w, f^{\prime} \not \models \Gamma$. So, there is $\psi ; \mathbf{t} \in \Gamma$ such that $w, f^{\prime}(\mathbf{t}) \models \neg \psi$. Now, by the restriction on the deduction rule, s properly extends $\mathbf{t}$. But then, $f^{\prime}(\mathbf{t})=$ $f(\mathbf{t})$, but $w, f(\mathbf{t}) \models \psi$.

Now, since each deduction rule is sound, it is simple to show the result by induction on lengths of deductions.

Finally, a soundness result can be given for the kind of entailments which are of principal importance - those between unlabelled formulas (since entailment between labelled formulas is supposed to be purely instrumental):

Proposition 3 Let $\Gamma$ be a set of formulas of $\mathscr{L}_{\downarrow}$ and $\phi$ a formula of $\mathscr{L}_{\downarrow}$. Then:

$$
\text { If } \Gamma \vdash \phi \text { then } \Gamma \models \phi
$$

Proof All that is needed for the proof is that if $\Gamma^{*} \models \phi ;-$, then $\Gamma \models \phi$ (where $\Gamma^{*}$ is as in Definition 5). Suppose that $\Gamma^{*} \models \phi$; - . Consider some $w \in W_{\mathscr{M}}$ such that $\langle w\rangle \models \psi$ for each $\psi \in \Gamma$. Then clearly $w, f(\mathbf{s}) \models \psi$ for each $\psi, \mathbf{s} \in \Gamma^{*}$, since $\mathbf{s}$, and so $f(\mathbf{s})$ is empty in each case. So $w, f(\langle\rangle) \models \phi$. I.e. $\langle w\rangle \models \phi$ as required.

The soundness result then follows easily.

\subsection{Completeness}

The completeness of the basic logic for serial frames is relatively simple and can be proved along familiar lines. It needs to be shown that for any set of formulas $\Gamma$, if $\Gamma$ is consistent (i.e. $\Gamma \nvdash \perp$ ) then it is satisfiable (i.e. there are $\mathscr{M}, w \in W_{\mathscr{M}}$ such that $\mathscr{M},\langle w\rangle \models \Gamma)$.

By the usual reasoning, there is a maximally consistent set of labelled formulas $\Gamma^{\prime}$ such that $\Gamma^{*} \subseteq \Gamma^{\prime}$ (where, recall, $\Gamma^{*}=\{\phi ;-: \phi \in \Gamma\}$ ). As in the proofs of completeness for predicate logic, some care is needed in the construction of $\Gamma^{\prime}$ so that if $\diamond \phi ; \mathbf{s} \in \Gamma^{\prime}$, then for some $n \in \mathbb{N}, \phi ;\langle\mathbf{s}, n\rangle \in \Gamma^{\prime}$. This is analogous to the care needed in first-order logic to ensure that if $\exists x \phi$ appears in the maximally consistent set, then a witness appears as well.

$\Gamma^{\prime}$ is then satisfiable in the sense that there are $\mathscr{M}, w \in W_{\mathscr{M}}$ and $f \in \operatorname{Hom}_{\mathscr{M}}$ such that $\mathscr{M}, w, f \models \Gamma^{\prime}$. Let $\mathscr{M}=\langle W, R, a\rangle$ such that $W=\mathbb{N}^{<\omega}, \mathbf{s} R \mathbf{t}$ iff $\mathbf{t}=\langle\mathbf{s}, n\rangle$ for some $n \in \mathbb{N}$, and $a(p, \mathbf{s})=T$ iff $p ; \mathbf{s} \in \Gamma^{\prime}$. Now let $f \in$ Hom $_{\mathscr{M}}$ be such that $f\left(\left\langle n_{1}, \ldots, n_{k}\right\rangle\right)=\left\langle\left\langle n_{1}\right\rangle,\left\langle n_{1}, n_{2}\right\rangle, \ldots,\left\langle n_{1}, \ldots, n_{k}\right\rangle\right\rangle$.

Then, it is easy to check that $\mathscr{M},\langle\rangle, f \models \Gamma^{\prime}$. From this it is easy to see that $\mathscr{M},\langle\langle\rangle\rangle \mid=\Gamma$.

Now, from the result that consistent sets of formulas are satisfiable, it is simple to prove (in the usual way) that if $\Gamma \models \phi$, then $\Gamma \vdash \phi$. 


\section{Strengthenings}

In this section, I will consider how the logic may be strengthened by the addition of rules which correspond to usual restrictions on the accessibility relation. So, I will consider extensions to the systems T, S4 and S5, which model theoretically correspond to restricting consideration to models whose accessibility relation is reflexive, transitive and reflexive, and an equivalence relation, respectively.

In terms of the semantics, we can make restrictions on allowable accessibility relations in the usual way. So, for example, we can require that the relation is reflexive (resulting in the system T), transitive and reflexive (resulting in the system S4), or restrict attention to equivalence relations (resulting in S5, which could also be achieved by doing away with the relation altogether). The latter of these brings us much closer to the kind of system which Hodes considered when introducing the backtracking operator. Other restrictions on the relation could also be considered, but I will restrict attention to these three here.

The standard way of reflecting these restrictions in the proof theory are not, however, suitable in the presence of the backtracking operator. At least, not without modification. So, for example, suppose that we add the following schema:

$$
\square \phi \rightarrow \square \square \phi
$$

Or, alternatively, strengthen the inference rules so that (4) is provable (cf. [4, ch. IV], [5]).

Then we can instantiate $\phi$ with $\downarrow \psi$, for any formula $\psi$. The result is:

$$
\square \downarrow \psi \rightarrow \square \square \downarrow \psi .
$$

But then, from this, it clearly follows that:

$$
\psi \rightarrow \square \psi,
$$

resulting in a modal collapse.

Likewise, the usual (T) schema:

$$
\square \phi \rightarrow \phi,
$$

will also cause problems. If we add its necessitation to the system, or strengthen the rules so that it is provable, then we will have as an instance:

$$
\square(\square \downarrow \psi \rightarrow \downarrow \psi),
$$

which can be seen to entail

$$
\diamond \psi \rightarrow \psi
$$

again resulting in a modal collapse.

Finally, the usual (5) schema:

$$
\diamond \square \phi \rightarrow \square \phi
$$

has as an instance:

$$
\diamond \square \downarrow \psi \rightarrow \square \downarrow \psi
$$

which clearly entails:

$$
\diamond \psi \rightarrow \psi
$$


In order to add such axioms or strengthened rules, restrictions must be added on the kind of formulas which define instances. ${ }^{4}$

First, we define the degree of a formula, which measures the depth of nested backtracking operators which have not been cancelled by a modal operator. The resulting definition is equivalent to one given by $[1, \mathrm{p} .426]^{5}$ but we shall go via a more general definition (which will be useful later on).

Definition 10 Let $\phi$ be a formula of $\mathscr{L}_{\downarrow}$. Then $\operatorname{degs}(\phi) \subset \mathbb{N}$ is defined inductively as follows:

- $\quad$ For atomic $p, \operatorname{degs}(p)=\{0\}$

- $\operatorname{degs}(\neg \phi)=\operatorname{degs}(\phi)$

- $\operatorname{degs}(\phi \wedge \psi)=\operatorname{degs}(\phi) \cup \operatorname{degs}(\psi)$

- $\operatorname{degs}(\square \phi)=\{n-1: n \in \operatorname{degs}(\phi)\}$

- $\operatorname{degs}(\downarrow \phi)=\{n+1: n \in \operatorname{degs}(\phi)\}$

where $n-m=\max (n-m, 0)$. Then define the degree of $\phi, \operatorname{deg}(\phi)=\max (\operatorname{degs}(\phi))$.

The following lemma will then be useful:

Lemma 1 Let $\phi$ be any formula and $\mathscr{M}$ a model. Then for any finite sequences of worlds $\left\langle w_{n}, \ldots, w_{0}\right\rangle,\left\langle w_{m}^{\prime}, \ldots, w_{0}^{\prime}\right\rangle$ (not necessarily in $\mathrm{WS}_{\mathscr{M}}$ ) such that:

- $\quad n, m \geq \operatorname{deg}(\phi)$ and

- $\quad w_{i}=w_{i}^{\prime}$ for all $i \in \operatorname{degs}(\phi)$,

then:

$$
\left\langle w_{n}, \ldots, w_{0}\right\rangle \models \phi \text { iff }\left\langle w_{m}^{\prime}, \ldots, w_{0}^{\prime}\right\rangle \models \phi
$$

Proof By induction on formula complexity.

The base case, where $\phi=p$ is an atomic formula, is simple. Then $\operatorname{degs}(p)=\{0\}$, and:

$$
\begin{aligned}
\left\langle w_{n}, \ldots, w_{0}\right\rangle \models p & \Leftrightarrow a\left(w_{0}, p\right)=T & & (\text { Definition 2) } \\
& \Leftrightarrow a\left(w_{0}^{\prime}, p\right)=T & & \left(\text { Since } w_{0}=w_{0}^{\prime}\right) \\
& \Leftrightarrow\left\langle w_{m}^{\prime}, \ldots, w_{0}^{\prime}\right\rangle \models p & & (\text { Definition 2) }
\end{aligned}
$$

\footnotetext{
${ }^{4}$ Another approach to restricting these axioms is given by [3, Appendix 1.2] for a slightly different language. Parsons' approach is not however readily applicable to the language featuring $\downarrow$. His language features scoping operators which, instead of exempting a subformula from some fixed number of modal operators, signify that some subformula falls under the scope of some fixed number of modal operators.

${ }^{5}$ Hodes uses his definition to restrict the definition of logical consequence, rather than for the purposes of restricting axioms and rules.
} 
Now, suppose that $\phi$ is $\psi \wedge \theta$, so that $\operatorname{degs}(\phi)=\operatorname{degs}(\psi) \cup \operatorname{degs}(\theta)$. Then:

$$
\begin{aligned}
& \left\langle w_{n}, \ldots, w_{0}\right\rangle \models \psi \wedge \theta \\
& \Leftrightarrow\left\langle w_{n}, \ldots, w_{0}\right\rangle \models \psi \text { and }\left\langle w_{n}, \ldots, w_{0}\right\rangle \models \theta \\
& \Leftrightarrow\left\langle w_{m}^{\prime}, \ldots, w_{0}^{\prime}\right\rangle \models \psi \text { and }\left\langle w_{m}^{\prime}, \ldots, w_{0}^{\prime}\right\rangle \models \theta \quad(\operatorname{since} \operatorname{degs}(\psi), \operatorname{degs}(\theta) \subseteq \operatorname{degs}(\phi) \\
& \Leftrightarrow\left\langle w_{m}^{\prime}, \ldots, w_{0}^{\prime}\right\rangle \models \psi \wedge \theta
\end{aligned}
$$

The proof is similar when $\phi$ is $\neg \psi$.

Suppose that $\phi$ is $\square \psi$. First, note that, if $i+1 \in \operatorname{degs}(\psi)$, then $i \in \operatorname{degs}(\square \phi)$, and thus that $w_{i}=w_{i}^{\prime}$. We then have that:

$$
\left\langle w_{n}, \ldots, w_{0}\right\rangle \models \square \phi
$$

$\Leftrightarrow \quad$ For all $u$ s.t. $w_{0} R u,\left\langle w_{n}, \ldots, w_{0}, u\right\rangle \models \phi$

(Definition 2)

$\Leftrightarrow \quad$ For all $u$ s.t. $w_{0} R u,\left\langle w_{m}^{\prime}, \ldots, w_{0}^{\prime}, u\right\rangle \models \phi$

(Inductive hypothesis and the above observation)

We then need to show that this last line is equivalent to:

$$
\text { For all } u^{\prime} \text { s.t. } w_{0}^{\prime} R u^{\prime},\left\langle w_{m}^{\prime}, \ldots, w_{0}^{\prime}, u^{\prime}\right\rangle \models \phi
$$

If $0 \notin \operatorname{degs}(\phi)$, this follows from the inductive hypothesis. If $0 \in \operatorname{degs}(\phi)$, then $w_{0}=w_{0}^{\prime}$, and so this trivially follows.

Finally, suppose that $\phi$ is $\downarrow \psi$. Note that if $i \in \operatorname{degs}(\psi)$ then $i+1 \in \operatorname{degs}(\downarrow \psi)$. Then:

$$
\begin{aligned}
\left\langle w_{n}, \ldots, w_{0}\right\rangle & \models \downarrow \psi \\
\Rightarrow \quad\left\langle w_{n}, \ldots, w_{1}\right\rangle & \models \psi \\
\Rightarrow \quad\left\langle w_{n}^{\prime}, \ldots, w_{1}^{\prime}\right\rangle & \models \psi \quad \text { (Inductive hypothesis and the above observation) } \\
\Rightarrow \quad\left\langle w_{n}^{\prime}, \ldots, w_{0}^{\prime}\right\rangle & \models \downarrow \psi
\end{aligned}
$$

as required.

We are now in a position to strengthen the logic. This could either be done by adding axioms, or by strengthening rules. Since it fits in better with the natural deduction style of the proof theory so far, I will consider strengthening rules, though not much hangs on this. ${ }^{6}$

To strengthen the logic to (T), we strengthen the ( $\square$-E) rule to:

$$
\left(\square-\mathrm{E}^{\prime}\right) \frac{\square \phi ; \mathbf{s}}{\phi ; \mathbf{s}}
$$

with a restriction that $\operatorname{deg}(\phi)=0$.

There will also be a corresponding derived rule for $\diamond$ :

$$
\left(\diamond-I^{\prime}\right) \frac{\phi ; \mathbf{s}}{\diamond \phi ; \mathbf{s}}
$$

\footnotetext{
${ }^{6}$ These strengthenings of rules are all closely related to the rules in [5], albeit using labels in place of strict subproofs, and with restrictions related to degrees.
} 
again, with a restriction to degree 0 formulas. It is easy to check that this is derivable from $\square$-E. It is also simple to prove the (T) axiom (restricted to formulas with degree 0 ) from this rule.

To strengthen the logic to S4, we add another rule for necessitation, called necessitation reiteration:

with a restriction that $\operatorname{deg}(\phi)=0$.

$$
(\square-\mathrm{R}) \frac{\square \phi ; \mathbf{s}}{\square \phi ;\langle\mathbf{s}, n\rangle}
$$

Again, there is a corresponding derived rule for $\diamond$ :

$$
\left(\diamond-\mathrm{R}^{-}\right) \frac{\diamond \phi ; \mathbf{s}}{\nabla \phi ; \mathbf{s}^{-}}
$$

with the same restriction. (The superscript on the rule name will become apparent.) And from this rule, we can easily prove the (4) axiom (restricted to formulas with degree 0).

Finally, we can add another reiteration rule to strengthen the logic to S5:

$$
\left(\diamond-\mathrm{R}^{+}\right) \frac{\diamond \phi ; \mathbf{s}}{\diamond \phi ;\langle\mathbf{s}, n\rangle}
$$

with the same restriction again. From this we can derive the usual (5) axiom schema (again, restricted).

\subsection{Soundness}

These rules are sound with respect to models whose equivalence relations are reflexive, transitive and reflexive, and equivalence relations, respectively. In each case, the proof proceeds by use of Lemma 1 .

Proposition 4 Suppose that $\mathscr{M}$ is such that $R_{\mathscr{M}}$ is reflexive, $f$ is a homomorphism, and $w \in W_{\mathscr{M}}$ a world. Then, for any $\phi$ with $\operatorname{deg}(\phi)=0$, if $\mathscr{M}, w, f \models \square \phi$; s, then $\mathscr{M}, w, f \models \phi ; \mathbf{s}$.

Proof Suppose that $w, f \models \square \phi$; $\mathbf{s}$, so that $\langle w, f(\mathbf{s})\rangle \models \square \phi$. Let $w^{\prime}=t(f(\mathbf{s}))$. Then, by Lemma $1,\left\langle w^{\prime}\right\rangle \models \square \phi$.

By the semantic clause for $\square$, for any $w^{\prime \prime}$ such that $w^{\prime} R w^{\prime \prime},\left\langle w^{\prime}, w^{\prime \prime}\right\rangle \models \phi$. Since $R$ is reflexive $w^{\prime} R w^{\prime}$, and so $\left\langle w^{\prime}, w^{\prime}\right\rangle \models \phi$. Then, by Lemma $1,\langle w, f(\mathbf{s})\rangle \models \phi$, as required.

Proposition 5 Suppose that $\mathscr{M}$ is such that $R_{\mathscr{M}}$ is transitive, $f$ is a homomorphism, and $w \in W_{\mathscr{M}}$ a world. Then, for any $\phi$ with $\operatorname{deg}(\phi)=0$, if $\mathscr{M}, w, f \models \square \phi$; s, then $\mathscr{M}, w, f \models \square \phi ;\langle\mathbf{s}, n\rangle$.

Proof Assume that $\langle w, f(\mathbf{s})\rangle \models \square \phi$, and we aim to show that for any $n \in \mathbb{N}$, $\langle w, f(\langle\mathbf{s}, n\rangle)\rangle \models \square \phi$. By Proposition 1.1, and the definition of $\square$, we are required to show that, for any $w^{\prime}, w^{\prime \prime}$ such that $t(f(\mathbf{s})) R w^{\prime}$ and $w^{\prime} R w^{\prime \prime},\left\langle w, f(\mathbf{s}), w^{\prime}, w^{\prime \prime}\right\rangle \models \phi$.

But, since $\operatorname{deg}(\phi)=0$, and by Lemma 1 , this is equivalent to $\left\langle w, f(\mathbf{s}), w^{\prime \prime}\right\rangle \models \phi$. By transitivity, $t(f(\mathbf{s})) R w^{\prime \prime}$, so $\left\langle w, f(\mathbf{s}), w^{\prime \prime}\right\rangle=\phi$ by our original assumption and the definition of $\square$, as required. 
Proposition 6 Suppose that $\mathscr{M}$ is such that $R_{\mathscr{M}}$ is transitive and symmetric, $f$ is a homomorphism, and $w \in W_{\mathscr{M}}$ a world. Then, for any $\phi$ with $\operatorname{deg}(\phi)=0$, if $\mathscr{M}, w, f \models \diamond \phi ; \mathbf{s}$, then $\mathscr{M}, w, f \models \diamond \phi ;\langle\mathbf{s}, n\rangle$.

Proof Assume that $w, f \models \diamond \phi$; s. So, there is $u \in W$ such that $t(f(\mathbf{s})) R u$ and $\langle w, f(\mathbf{s}), u\rangle \models \phi$. By Lemma $1,\langle u\rangle \models \phi($ since $\operatorname{deg}(\phi)=0)$.

Then to show that $w, f=\left\langle\phi ;\langle\mathbf{s}, n\rangle\right.$, we need to show that there is $u^{\prime} \in W$ such that $t(f(\langle\mathbf{s}, n\rangle)) R u^{\prime}$ and $\left\langle w, f(\langle\mathbf{s}, n\rangle), u^{\prime}\right\rangle \models \phi$. By Lemma 1, for the latter of these it is sufficient to show that $\left\langle u^{\prime}\right\rangle \models \phi$.

But then $u$ is such a $u^{\prime}$. We already have that $\langle u\rangle \models \phi$. The accessibility properties follow from the transitivity and symmetry of $R$, together with the fact that $f(\langle\mathbf{s}, n\rangle)=\left\langle f(\mathbf{s}), u^{\prime \prime}\right\rangle$ where $t(f(\mathbf{s})) R u^{\prime \prime}$.

Soundness theorems for appropriately restricted notions of logical consequence follow easily.

\subsection{Normal Forms and Degree Separation}

Before proving completeness for these strengthenings, it will be useful to prove that any formula is equivalent to one in which the degrees are 'separated', in a certain sense. To make this more precise, we have the following definition:

Definition 11 A formula $\phi$ is in degree-separated normal form (DSNF) iff it has the form:

$$
\bigwedge_{i=0}^{n} \phi_{i}
$$

where for each $i \leq n$, we have one of the following:

- $\operatorname{degs}\left(\phi_{i}\right)=\{0\}$,

- $0 \notin \operatorname{degs}\left(\phi_{i}\right)$, or

- $\phi_{i}$ is of the form $\psi \vee \theta$ where $\operatorname{degs}(\psi)=\{0\}$ and $0 \notin \operatorname{degs}(\theta)$.

So, a formula in DSNF is a conjuction of disjuctions, none of the disjuncts of which have both 0 and $n>0$ in their degrees. This is thus similar in some ways to conjunctive normal form in non-modal propositional logic, but where the role of atoms is played by formulas $\phi$ where either $\operatorname{deg}(\phi)=0$ or $0 \notin \operatorname{degs}(\phi)$. There is no need to have more than two disjuncts in each disjunction, since the disjuction of formulas of degree 0 will still be of degree 0 , and the disjunction of formulas with degree not including 0 will still not include degree 0 .

We wish to prove that each formula is equivalent to one in DSNF, and we can do so for a stronger notion of equivalence than we currently have. So, we define:

Definition 12 Two formulas $\phi$ and $\psi$ are equivalent, $\phi \equiv \psi$, iff for all models $\mathscr{M}$ and all non-empty $\mathbf{w} \in W_{\mathscr{M}}^{<\omega}$ (and not just in $\mathrm{WS}_{\mathscr{M}}$ ):

$$
\mathscr{M}, \mathbf{w} \models \phi \Leftrightarrow \mathscr{M}, \mathbf{w} \models \psi
$$


(Note that here we are appealing to the extended notion of satisfaction alluded to in Definition 2.)

Note that this is a stronger condition than $\models \phi \leftrightarrow \psi$, since, for example, $\models p \leftrightarrow$ $\downarrow p$ but $p \not \equiv \downarrow p$.

Before proving that every formula is equivalent to a DSNF formula, a couple of Lemmas will be useful:

Lemma 2 Suppose that $0 \notin \operatorname{degs}(\phi)$. Then $\diamond \phi \equiv \square \phi$.

Proof The right to left direction follows from the assumption that the accessibility relation is serial.

For the left to right direction, suppose that $\mathbf{w} \models \diamond \phi$. Then, for some $u$ with $t(\mathbf{w}) R u,\langle\mathbf{w}, u\rangle \models \phi$. But then, by Lemma 1 , for any $u$ with $t(\mathbf{w}) R u,\langle\mathbf{w}, u\rangle \models \phi$, and thus $\mathbf{w} \models \square \phi$ as required.

Lemma 3 Suppose that $\operatorname{degs}(\phi)=\{0\}$ and $0 \notin \operatorname{degs}(\psi)$. Then $\square(\phi \vee \psi) \equiv \square \phi \vee$ $\square \psi$.

Proof The right to left direction is trivial.

For the left to right direction of the equivalence, suppose that $\mathbf{w} \models \square(\phi \vee \psi)$. Suppose that $\mathbf{w} \not \models \square \phi$, then there is $u$ with $t$ (w) $R u$ such that $\langle\mathbf{w}, u\rangle \not \models \phi$ but $\langle\mathbf{w}, u\rangle \models$ $\phi \vee \psi$, and thus $\langle\mathbf{w}, u\rangle \models \psi$. Thus, $\mathbf{w} \models \diamond \psi$. Then, by Lemma $2,\langle\mathbf{w}\rangle \models \square \psi$. Hence $\langle\mathbf{w}\rangle \models \square \phi \vee \square \psi$ as required.

We can now prove that every formula is equivalent to one in DSNF:

Proposition 7 Every formula $\phi$ is a equivalent to a DSNF formula $\phi^{\prime}$ such that $\operatorname{degs}(\phi)=\operatorname{degs}\left(\phi^{\prime}\right)$.

Proof By induction on formula complexity. The base case is trivial since atomic propositions are already in DSNF. Likewise, the inductive step for $\wedge$ is trivial.

The inductive step for $\neg$ is simple, following from basic applications of de Morgan's laws and the distributivity of conjunction and disjunction, and a simple observation that the resulting formula has the right form.

The inductive step for $\downarrow$ follows from the fact that $\downarrow$ distributes over both conjunction and disjunction.

For the case where $\phi$ is $\square \psi$ :

$$
\begin{aligned}
\phi & =\square \psi \\
& \equiv \square \bigwedge \psi_{i} \quad \text { (By the inductive hypothesis) } \\
& \equiv \bigwedge \square \psi_{i}
\end{aligned}
$$

When $\psi$ is a disjunction of a formula of degree 0 and a formula of degree not including 0 , then $\square$ will distribute over this, resulting in a formula of the correct form. 


\subsection{Completeness}

We are now in a position to prove completeness theorems corresponding to the soundness theorems above. We will do so by showing that the models constructed in the completeness proof for the unstrengthened system can be extended to models which satisfy the required restrictions on the accessibility relation.

\subsubsection{Reflexive models}

So, for the case of reflexivity, we need to prove the following theorem:

Proposition 8 Suppose that $\mathscr{M}=\langle W, R, a\rangle \models T$, but $R$ is not reflexive. Let $\mathscr{M}^{\prime}=$ $\left\langle W, R^{\prime}, a\right\rangle$, where $R^{\prime}$ is such that, for all $w, w^{\prime} \in W, w R^{\prime} w^{\prime}$ iff $w R w^{\prime}$ or $w=w^{\prime}$.

Then, for any formula $\phi$, and any $\mathbf{w} \in \mathrm{WS} \mathscr{M}$ :

$$
\mathscr{M}, \mathbf{w} \models \phi \text { iff } \mathscr{M}^{\prime}, \mathbf{w} \models \phi .
$$

The proof will be by induction on formula complexity. Since the only difference between the two models is the accessibility relation, the base case (atomic propositions) and all the inductive steps other than for necessity are trivial.

The inductive step for formulas of the form $\square \phi$ is more tricky. What we will need to show is that, on the assumption that Proposition 8 holds for $\phi$, then for any $\mathbf{w} \in \mathrm{WS}_{\mathscr{M}}$ :

$$
\mathscr{M}, \mathbf{w} \models \square \phi \text { iff } \mathscr{M}^{\prime}, \mathbf{w} \models \square \phi .
$$

Since $R$ is a subrelation of $R^{\prime}$, the right to left direction is trivial, and it is easy to see that for the left to right direction it is sufficient to show that:

$$
\text { If } \mathscr{M}, \mathbf{w} \models \square \phi \text { then } \mathscr{M}^{\prime},\langle\mathbf{w}, t(\mathbf{w})\rangle \models \phi \text {. }
$$

We will show this first for DSNF formulas, and then use Proposition 7 to prove it in general. In fact, we shall prove a slightly modified version of the result, which does not require assuming the inductive hypothesis.

Proposition 9 Suppose that $\mathscr{M} \models T$, and that $\phi$ is in DSNF. Then, for all $\mathbf{w} \in$ WS $\mathscr{M}$ :

$$
\text { If } \mathscr{M}, \mathbf{w} \models \square \phi \text { then } \mathscr{M},\langle\mathbf{w}, t(\mathbf{w})\rangle \models \phi .
$$

(Note that it is $\mathscr{M}$, and not $\mathscr{M}^{\prime}$, which appears in the consequent, and that there is no guarantee that $\langle\mathbf{w}, t(\mathbf{w})\rangle \in \mathrm{WS} \mathscr{M}$.)

Proof First, we can prove the result for when $\operatorname{degs}(\phi)=\{0\}$. Suppose that $\mathscr{M}, \mathbf{w} \models$ $\square \phi$. Then, since $\mathscr{M} \models T$ and $\operatorname{deg}(\phi)=0, \mathscr{M}, \mathbf{w} \models \phi$. Then, by Lemma 1 , $\mathscr{M},\langle\mathbf{w}, t(\mathbf{w})\rangle \models \phi$ as required.

Secondly, we can prove when $0 \notin \operatorname{degs}(\phi)$. Suppose that $\mathscr{M}, \mathbf{w}=\square \phi$. Then, for all $u$ with $t(\mathbf{w}) R u, \mathscr{M},\langle\mathbf{w}, u\rangle \models \phi$, and by seriality there is such a $u$. Hence, by Lemma 1 , and since $0 \notin \operatorname{degs}(\phi), \mathscr{M},\langle\mathbf{w}, t(\mathbf{w})\rangle \models \phi$ as required.

Then, we can prove it for when $\phi$ is of the form $\psi \vee \theta$, where $\operatorname{degs}(\psi)=\{0\}$ and $0 \notin \operatorname{degs}(\theta)$. Suppose that $\mathscr{M}, \mathbf{w} \models \square\left(\phi_{1} \vee \phi_{2}\right)$. Then, by Lemma $3, \mathscr{M}, \mathbf{w} \models$ 
$\square \phi_{1} \vee \square \phi_{2}$. Then, by the previous two results, we get that $\mathscr{M},\langle\mathbf{w}, t(\mathbf{w})\rangle \models \phi$ as required.

Finally, this extends to all formulas in DSNF, since necessity distributes over conjunctions.

Finally, we can prove Proposition 8:

Proof of Proposition 8 As mentioned, the base case and all inductive steps are trivial except for necessity.

So, suppose that the proposition holds for $\phi$ and we wish to show it for $\square \phi$. We need to show that:

$$
\text { If } \mathscr{M} \text {, w } \models \square \phi \text { then } \mathscr{M}^{\prime},\langle\mathbf{w}, t(\mathbf{w})\rangle \models \phi
$$

By Proposition 7, we have that there is $\phi^{\prime} \equiv \phi$ which is in DSNF. Then:

$$
\begin{aligned}
\mathscr{M}, \mathbf{w}=\square \phi & \Rightarrow \mathscr{M}, \mathbf{w}=\square \phi^{\prime} & & \left(\phi \equiv \phi^{\prime}\right) \\
& \Rightarrow \mathscr{M},\langle\mathbf{w}, t(\mathbf{w})\rangle \models \phi^{\prime} & & (\text { Lemma } 9) \\
& \Rightarrow \mathscr{M},\langle\mathbf{w}, t(\mathbf{w})\rangle \models \phi & & \left(\phi \equiv \phi^{\prime}\right) \\
& \Rightarrow \mathscr{M}^{\prime},\langle\mathbf{w}, t(\mathbf{w})\rangle \models \phi & & \text { (Inductive hypothesis) }
\end{aligned}
$$

as required. The proposition then follows.

Completeness is then trivial. Given a consistent set of formulas, we can construct a model as in Section 5, and then allow its accessibility relation to be reflexive. This will then be a model of $\Gamma$, by Proposition 8 .

\subsubsection{Transitive models and equivalence relation models}

The argument for transitive models and equivalence relation models is much the same. In both cases, we again replace the accessibility relation of a model with one which is transitive or an equivalence relation in the same kind of way. So, to get a transitive model, we take the transitive closure of the relation, and to get an equivalence relation, we let $w_{1} R^{\prime} w_{2}$ iff $w_{1}=w_{2}, w_{1} R^{*} w_{2}$ or $w_{2} R^{*} w_{1}$, where $R^{*}$ is the transitive closure of $R$.

In each case, it is again only the step corresponding to necessity in a proof by induction which is problematic. Note that, in the previous case, the only point at which we appealed to the features of the new accessibility relation (rather than appealing to Lemma 1, which is general), is in proving the step for formulas with degree 0 . That can be done again here.

Proposition 10 Suppose that $\mathscr{M}=\langle W, R, a\rangle \models 4, \phi \in \mathscr{L}_{\downarrow}$ such that $\operatorname{deg}(\phi)=0$ and let $R^{\prime}$ be the transitive closure of $R$. Then, for all $\mathbf{w} \in \mathrm{WS}_{\mathscr{M}}$, if $\mathscr{M}, \mathbf{w} \models \square \phi$, then for all $u \in W$ such that $t(\mathbf{w}) R^{\prime} u, \mathscr{M},\langle\mathbf{w}, u\rangle \models \phi$.

Proof Suppose that $\mathbf{w} \models \square \phi$, and let $u$ be such that $t(\mathbf{w}) R^{\prime} u$. Then, there are $u_{1}, \ldots, u_{n}$ such that $t(\mathbf{w}) R u_{1}, u_{1} R u_{2}, \ldots, u_{n} R u$. 
We then have:

$$
\begin{aligned}
\mathbf{w} \models \square \phi & \Rightarrow \mathbf{w} \models \underbrace{\square \ldots \square}_{n+1} \phi & & (\text { Since } \mathscr{M} \models 4) \\
& \Rightarrow\left\langle\mathbf{w}, u_{1}, \ldots, u_{n}, u\right\rangle \models \phi & & \text { (Definition 2) } \\
& \Rightarrow\langle\mathbf{w}, u\rangle \models \phi & & \text { (Lemma 1) }
\end{aligned}
$$

as required.

Proposition 11 Suppose that $\mathscr{M}=\langle W, R, a\rangle \models S 5, \phi \in \mathscr{L}_{\downarrow}$ such that $\operatorname{deg}(\phi)=0$ and let $R^{\prime}$ be the smallest equivalence relation including $R$.

Then, for all $\mathbf{w} \in \mathrm{WS}_{\mathscr{M}}$, if $\mathscr{M}, \mathbf{w} \models \square \phi$, then for all $u \in W$ such that $t(\mathbf{w}) R^{\prime} u$, $\mathscr{M},\langle\mathbf{w}, u\rangle \models \phi$.

Proof First note that for any $w_{1}, w_{2}, w_{1} R w_{2}$ iff one of the following holds:

- $\quad w_{1}=w_{2}$

- $w_{1} R^{*} w_{2}$

- $w_{2} R^{*} w_{1}$

where $R^{*}$ is the transitive closure of $R$.

Suppose that $\mathbf{w} \models \square \phi$, and let $u$ be such that $t(\mathbf{w}) R^{\prime} u$. We want to show that $\langle\mathbf{w}, u\rangle \models \phi$. We have already proved this in the cases where $u=t(\mathbf{w})$ and where $t(\mathbf{w}) R^{*} u$.

The only case left is where $u R^{*} t(\mathbf{w})$. So, there are $u_{1}, \ldots, u_{n} \in W_{\mathscr{M}}$ such that $u R u_{1}, u_{1} R u_{2}, \ldots, u_{n} R t(\mathbf{w})$.

Suppose for contradiction that $\langle\mathbf{w}, u\rangle \not \models \phi$. Then:

$$
\begin{array}{rlrl} 
& \langle\mathbf{w}, u\rangle \models \neg \phi & & \text { (Definition 2) } \\
\Rightarrow & \langle u\rangle \models \neg \phi & & \text { (Lemma 1) } \\
\Rightarrow & \langle u\rangle \models \diamond \neg \phi & & \text { (Since } \mathscr{M} \models T \text { ) } \\
\Rightarrow & \langle u\rangle \models \underbrace{\square}_{n+1} \ldots \diamond \neg \phi & & \text { (Since } \mathscr{M} \models 4 \text { and } \mathscr{M} \models 5 \text { ) } \\
\Rightarrow & \left\langle u, u_{1}, \ldots, u_{n}, t(\mathbf{w})\right\rangle \models \diamond \neg \phi & & \text { (Definition 2) } \\
\Rightarrow \quad \mathbf{w} \models \diamond \neg \phi & & \text { (Lemma 1) }
\end{array}
$$

But this contradicts our assumption that $\mathbf{w} \models \square \phi$. Hence $\langle\mathbf{w}, u\rangle \models \phi$, as required.

The additional steps for the completeness proof can then be carried out in just the same way as for transitive models. 


\section{Conclusion}

I hope that a proof theory of the sort presented in this paper will be useful to those who would like to understand backtracking operators in a way which does not essentially involve possible worlds.

The proof system is motivated, not by possible worlds semantics, but rather by the interpretation of the operator as indicating scope. The proof theory is sound and complete with respect to the semantics. This means that the proof theory is acceptable to those who would rather interpret the operator semantically-so that the proof theory plays a useful role in showing that certain formulas follow (semantically) from others. It also means that the semantics can play a useful pragmatic role for those who interpret the operator more syntactically_-so that model-theoretic arguments can be given to show that certain inferences can or can not be made in the proof system.

Most philosophical uses of a backtracking operator-and in particular those uses to which a proof theory would be most useful - are in the context of quantified modal logic. But, although the approach here concerns only propositional logic, much of what I have said can readily be applied to the quantified case. For example, rules for the quantifiers can be added in the obvious way-simply take the usual rules for quantifiers, with the addition that they preserve the label on a formula (in just the same way as the rules for connectives here). Then the soundness proofs simply need an additional check for these rules, and the completeness proof will combine the completeness proof here with a typical proof of the completeness of predicate logic.

Acknowledgments Thanks to Bob Hale, Rosanna Keefe and Øystein Linnebo for useful comments on earlier drafts; the attendees at the 4th $\mathrm{PhDs}$ in Logic conference in Ghent, where I presented a version of the paper; and an anonymous referee. This work was supported by an Arts and Humanities Research Council postgraduate research award, and by a Jacobsen Fellowship at the University of London.

\section{References}

1. Hodes, H. (1984). On modal logics which enrich first-order S5. Journal of Philosophical Logic, 13, 423-454.

2. Linnebo, Ø. (2009). Bad company tamed. Synthese, 170(3), 371-391.

3. Parsons, C. (1983). Mathematics in Philosophy. In Sets and modality, (pp. 298-341): Cornell University Press.

4. Prawitz, D. (1965). In Natural Deduction: A Proof Theoretic Study. Mineola: Dover.

5. Siemens, D.F. (1977). Fitch-style rules for many modal logics. Notre Dame Journal of Formal Logic, 18(4), 631-636.

6. Uzquiano, G. (2011). Plural quantification and modality. Proceedings of the Aristotelian Society, 111(2), 219-250.

7. Uzquiano, G. (2014). Mereology and modality. In Kleinschmidt, S. (Ed.) Mereology and Location: Oxford University Press.

8. Williamson, T. (2010). Necessitism, contingentism, and plural quantification. Mind, 119(475), 657748 . 